peripheral or intraosseous line(s) and full non-invasive monitoring, ensuring patients are well oxygenated and have an adequate BP may also improve transfer times.

\section{BURTON PAEDIATRIC EARLY WARNING SYSTEM SCORE}

doi:10.1136/archdischild-2012-302724.1483

'M Ahmed, 'D Sobithadevi, 'R Lall, 'A Ghose, ${ }^{2} S$ Boswell, ${ }^{1} \mathrm{~T}$ Reynolds. 'Paediatrics, ${ }^{2}$ Paediatric Research Nurse; ${ }^{3}$ Clinical Chemistry, Burton Hospitals NHS Foundation Trust, Burton Upon Trent, UK

Background Early warning scores compliment clinical decision making and can identify trends depicting deterioration in patient's condition. Age appropriate Burton Paediatric Early Warning System (BPEWS) score charts were developed in 2011 using nine indicators which included physiological parameters, therapeutic intervention and doctor/nurse concern.

Aim To assess the usefulness of BPEWS as a reliable and valid indicator for all children in need of urgent medical assessment and intervention.

Methods A retrospective analysis of all children transferred to paediatric intensive care setting over the preceding 12 months was carried out to validate BPEWS charts. Detailed case notes review was undertaken to evaluate if BPEWS could have been useful to alert us of patients' deterioration in the 24 hour period prior to transfer. Each case note was assessed by two reviewers.

Results An average of 8.7 sets of observations per patient was recorded in the 24 hours period prior to intensive care transfer. Off the 200 sets of observations recorded in 23 patients, $93 \%$ sets would have triggered based on BPEWS. 44\% sets of observation scores were in amber (4-7) while 35\% were in red (>7) category. Average highest BPEWS score was 9.5 (range: $4-19$ ). In $43 \%$ and $57 \%$ of patients, highest BPEWS score fell in amber and red category respectively. Conclusions BPEWS score charts are effective in identifying children at risk of sudden deterioration. Timely identification is likely to enable early action to reduce the risk of death or serious morbidity thus improving the outcome of care given to hospitalised children.

\section{ACCURACY OF A SEQUENTIAL APPROACH TO IDENTIFY YOUNG FEBRILE INFANTS AT LOW RISK FOR INVASIVE BACTERIAL INFECTION}

doi:10.1136/archdischild-2012-302724.1484

1.2S Mintegi, ${ }^{3} S$ Bressan, 'B Gomez, ${ }^{4} \mathrm{~L}$ Da Dalt, ${ }^{5}$ Olaciregui, ${ }^{6} \mathrm{M}$ De La Torre, ${ }^{7} \mathrm{M}$ Palacios, ${ }^{4} \mathrm{P}$ Berlese, ${ }^{8} \mathrm{~A}$ Ruano, ${ }^{1 \mathrm{~J}}$ Benito. ${ }^{1}$ Pediatric Emergency, Cruces University Hospital; 'Department of Pediatrics, University of the Basque Country, Bilbao, ${ }^{3}$ Department of Pediatrics, University of Padova, Padova, Spain; ${ }^{4}$ Ospedale Ca'Foncello, Department of Pediatrics, Treviso, Italy; ${ }^{5}$ Pediatric Emergency, Donostia University Hospital, Donostia; ${ }^{6}$ Pediatric Emergency, Niño Jesús University Hospital, Madrid; ${ }^{7}$ Pediatric Emergency, Navarra University Complex, Pamplona; ${ }^{8}$ Pediatric Emergency, Basurto University Hospital, Bilbao, Spain

Introduction Nowadays it is possible to manage as outpatients selected young febrile infants with low risk criteria for serious bacterial infection. A sequential approach, evaluating, firstly, the appearance of the infant, secondly, the urinanalysis, and, finally, the results of the blood biomarkers, including procalcitonin, may identify better low risk febrile infants suitable for outpatient management.

Objective To assess the value of a sequential approach ("step by step") to febrile young infants in order to identify low risk patients suitable for outpatient management and compare it with other ones previously described (Rochester criteria and Lab-score).

Methods A retrospective comparison of three different approaches ("step by step", Lab-score and Rochester criteria) was made in 1123 febrile infants less than 3 months of age attended in seven European Pediatric Emergency Departments.
Results Of the 1123 infants (Invasive Bacterial Infection - IBI-, 48; $4.2 \%), 488$ (43.4\%) were classified as low risk criteria for IBI according to the "step by step" approach (vs 693-61.7\%-with the Labscore and $458-40.7 \%$ - with the Rochester criteria). The prevalence of IBI in the low-risk criteria patients was $0.2 \%(95 \%$ CI $0-0.6 \%)$ using the "step by step" approach (vs $0.7 \%-95 \%$ CI $0.1-1.3 \%$ with the Labscore and $1.1 \%-95 \%$ CI $0.1-2 \%$ - with Rochester). Using the "step by step" approach, 1 patient with IBI was not correctly classified $(2.0 \%$, CI95\% $0-6.12)$ vs 5 using the Labscore or Rochester $(10.4 \%$, CI95\% 1.76-19.04\%)

Conclusions A sequential approach to young febrile infants including procalcitonin identifies better patients more suitable for outpatient management.

\section{ENDOSCOPIC ASPECTS OF INHALED VEGETABLE FOREIGN BODIES IN CHILDREN}

doi:10.1136/archdischild-2012-302724.1485

J Buzarov. Institute for Respiratory Diseases in Children-Kozle, Skopje, FYR Macedonia

Inhaled foreign bodies are very serious problem in the pediatric pulmonology since they increase the rate of morbidity and mortality. Aim of this study was analysis of endoscopic changes caused by vegetable foreign bodies (VFB) in correlation with their long - standing in the bronchial tree.

Material and Methods In ten years period (2002-2011) inhaled foreign bodies were removed in 219 children (age $6 \mathrm{mths}-14 \mathrm{yrs}$ ), $60.27 \%$-male and $39.73 \%$-female. Most of the children $(57.50 \%)$ belong to the youngest group of age (1-2 yrs). The inhaled foreign bodies were from organic origin in 208 (94.97\%). Of these, 203 $(92.69 \%)$ were with vegetable origin. The most commonly found grains were peanuts $(57.14 \%)$. Inhaled foreign bodies were single object in $123(56.16 \%)$ while in $96(43.80 \%)$ they were multiple.

Results Endoscopically we found:Insignificant inflammation (some hours presence of VFB) in 48 (23.64\%)Significant inflammation - vulnerable mucous membrane (VFB with presence more than 3 days) in 78 (38.42\%) Severe inflammation - manifest inflammation (VFB more than 7 days presence) in 77 (37.93\%). In this group of children we detected:- granulomatous formations $57(82.60 \%)$ decubital changes 28 (40.57\%)- mucopurulent secretion 41 (59.42\%). Conclusion Severity of the endoscopic changes was closely correlated with the quality of the foreign body (vegetable ones), the period of lodgement and the age of the patients. Timely broncoscopic extirpation of VFB decreases the percentage of complications and represents the most successful and only logically carried out therapeutically procedure.

\section{VALIDATION OF ADVANCED PAEDIATRIC LIFE SUPPORT FORMULAS FOR WEIGHT CALCULATION IN A MULTI- ETHNIC POPULATION}

doi:10.1136/archdischild-2012-302724.1486

'C Seddon, 'L Lockett, ${ }^{2} \mathrm{~S}$ Dhanjal, 'M Eisenhut. 'Paediatrics; 'Luton \& Dunstable Hospital NHS Foundation Trust, Luton, UK

Background In 2011 the advanced life support group (alsg) of the United Kingdom introduced a new formula for calculation of weight from age for paediatric emergencies. We present the first study validating this formula in a multi-ethnic population and comparing its performance to the formula currently used by the European Resuscitation Council (ERC) and other formulas.

Methods Prospective audit of weight versus calculated weight comparing alsg formula with ERC guideline, the Luscombe, Argall and Best Guess formulas analysed for gender, age and ethnic groups. Results We included prospectively 599 children aged 1 month to 12 years of age. There were 157 Asian, 268 Caucasian and 174 